\title{
Multiphasic strain differentiation of atypical mycobacteria from elephant trunk wash
}

Kok-Gan Chan, Mun Fai Loke, Bee Lee Ong, Yan Ling Wong, Kar Wai Hong, Kian Hin Tan, Sargit Kaur, Hien Fuh Ng, MFA Abdul Razak, Yun Fong Ngeow

Background: Two non-tuberculous mycobacterial strains, UM_3 and UM_11, were isolated from the trunk wash of captive elephants in Malaysia. As they appeared to be identical phenotypes, they were investigated further by conventional and whole genome sequencebased methods of strain differentiation. Methods: Multiphasic investigations on the isolates included species identification with hsp65 PCR-sequencing, conventional biochemical tests, rapid biochemical profiling using API strips and the Biolog Phenotype Microarray analysis, protein profiling with liquid chromatography-mass spectrometry, repetitive sequencebased PCR typing and whole genome sequencing followed by phylogenomic analyses. Results: The isolates were shown to be possibly novel slow-growing schotochromogens with highly similar biological and genotypic characteristics. Both strains have a genome size of $5.2 \mathrm{Mbp}, \mathrm{G}+\mathrm{C}$ content of $68.8 \%$, one rRNA operon and 52 tRNAs each. They qualified for classification into the same species with their average nucleotide identity of $99.98 \%$ and tetranucleotide correlation coefficient of 0.99999 . At the subspecies level, both strains showed $98.8 \%$ band similarity in the Diversilab automated repetitive sequence-based PCR typing system, $96.2 \%$ similarity in protein profiles obtained by liquid chromatography mass spectrometry, and a genomic distance that is close to zero in the phylogenomic tree constructed with conserved orthologs. Detailed epidemiological tracking revealed that the elephants shared a common habitat eight years apart, thus, strengthening the possibility of a clonal relationship between the two strains. 
1 Multiphasic strain differentiation of atypical mycobacteria from elephant trunk wash

2

3 Kok Gan Chan ${ }^{\mathrm{a}}$, Mun Fai Loke ${ }^{\mathrm{b}}$, Bee Lee Ong ${ }^{\mathrm{c}}$, Yan Ling Wong ${ }^{\mathrm{b}}$, Kar Wai Honga ${ }^{\mathrm{a}}$, Kian Hin

4 Tan $^{\mathrm{a}}$, Sargit Kaur ${ }^{\mathrm{b}}$, Hien Fuh Ng ${ }^{\mathrm{b}}$, MFA Abdul Razak ${ }^{\mathrm{d}}$, Yun Fong Ngeow ${ }^{\mathrm{b}, \mathrm{e}}$

5

6 Division of Genetics and Molecular Biology, Institute of Biological Sciences, Faculty of

7 Science, University of Malaya, Kuala Lumpur, Malaysia ${ }^{a}$; Department of Medical Microbiology,

8 Faculty of Medicine, University of Malaya, Kuala Lumpur, Malaysiab; Faculty of Veterinary

9 Medicine, Universiti Malaysia Kelantan, Malaysiac; Department of Wildlife and National Parks,

10 Peninsular Malaysia, Kuala Lumpur, Malaysiad; Faculty of Medicine and Health Sciences,

11 Universiti Tunku Abdul Rahman, Malaysia ${ }^{\mathrm{e}}$.

13 Kok Gan Chan (kokgan@um.edu.my)

14 Mun Fai Loke (lokemunfai@um.edu.my)

15 Bee Lee Ong (ong_beelee2010@yahoo.com)

16 Yan Ling Wong (wongyanling@gmail.com)

17 Kar Wai Hong (sebastian_karwai@hotmail.com)

18 Kian Hin Tan (tankianhin@hotmail.com)

19 Sargit Kaur (sargitk3@gmail.com)

20 Hien Fuh Ng (hienfuh@hotmail.com)

21 MFA Abdul Razak (mfirdaus@wildlife.gov.my)

22 \#Address correspondence to Yun Fong Ngeow (Email: yunngeow@yahoo.com; address: Jalan

23 Sungai Long, Bandar Sungai Long, Cheras 43000 Kajang, Selangor, Malaysia) 


\section{Abstract}

Background: Two non-tuberculous mycobacterial strains, UM_3 and UM_11, were isolated from the trunk wash of captive elephants in Malaysia. As they appeared to be identical phenotypes, they were investigated further by conventional and whole genome sequence-based methods of strain differentiation.

Methods: Multiphasic investigations on the isolates included species identification with hsp65 PCR-sequencing, conventional biochemical tests, rapid biochemical profiling using API strips and the Biolog Phenotype Microarray analysis, protein profiling with liquid chromatographymass spectrometry, repetitive sequence-based PCR typing and whole genome sequencing followed by phylogenomic analyses.

Results: The isolates were shown to be possibly novel slow-growing schotochromogens with highly similar biological and genotypic characteristics. Both strains have a genome size of $5.2 \mathrm{Mbp}, \mathrm{G}+\mathrm{C}$ content of $68.8 \%$, one rRNA operon and 52 tRNAs each. They qualified for classification into the same species with their average nucleotide identity of $99.98 \%$ and tetranucleotide correlation coefficient of 0.99999. At the subspecies level, both strains showed 98.8\% band similarity in the Diversilab automated repetitive sequence-based PCR typing system, $96.2 \%$ similarity in protein profiles obtained by liquid chromatography mass spectrometry, and a genomic distance that is close to zero in the phylogenomic tree constructed with conserved orthologs. Detailed epidemiological tracking revealed that the elephants shared a common habitat eight years apart, thus, strengthening the possibility of a clonal relationship between the two strains. 
46 Discussion: There is a remarkable consistency in the results from different types of analysis used.

47 In this study, genomic data supported the reliability of conventional methods for the strain differentiation of non-tuberculous mycobacteria. Nevertheless, more genetic studies on NTMs from different sources, geographic areas and times are necessary to develop reliable genotyping methods applicable to all NTM species.

\section{Introduction}

Bacterial strains are genetic variants of a bacterial species or subspecies. Strain differentiation is usually carried out to establish clonal transmission in disease outbreaks, to confirm crossinfection in healthcare settings, or to study evolutionary diversity among bacteria. Traditionally, strains are differentiated within species by their biological properties, antigenic variations or susceptibility to bacteriophages. Since the advent of molecular biology, however, DNA-based methods have increasingly become the methods of choice for bacterial typing in modern laboratories. The more frequently used techniques include multilocus sequence typing (MLST), pulsed-field gel electrophoresis (PFGE), restriction fragment length polymorphism (RFLP) studies, and repetitive sequence-based PCR (rep-PCR) typing. These methods have been effectively used in the investigation of tuberculosis transmission and reactivation in human patients (Gori et al., 2005) and animals (Aranaz et al., 2010).

Unlike Mycobacterium tuberculosis which is an established pathogen in humans as well as a wide range of domestic, wild and captive animals (Aranaz et al., 2010), the non-tuberculous mycobacteria (NTM), also known as atypical mycobacteria, are mostly saprophytes in the natural environment, only occasionally implicated in disease. The investigation of a NTM disease often 
involves genotyping to establish a link with an environmental source of infection. Unfortunately, the large number of NTM species and subspecies and their variable typeability in different assays has made it difficult to find a common test for genotyping. The closest to a gold standard test appears to be PFGE which has been successfully used on both rapidly growing and slowly growing NTMs (Jagielski et al., 2014). The discriminatory power of newer methods has not been adequately studied. Nevertheless, RFLP analysis of insertion sequences such as IS1245 and IS1311 was found to be useful for the differentiation of M. avium subspecies isolated from animal and human sources (Mijs et al., 2002). Similarly, variable number tandem repeat (VNTR) typing methods have been applied successfully in $M$. avium and $M$. avium subsp. paratuberculosis (Thibault et al., 2007) and have been used to confirm clonal relationships between patient and environmental isolates of M. ulcerans (Lavender et al., 2008). MLST and randomly amplified polymorphic DNA PCR (RAPD) have facilitated investigations of NTM outbreaks in laboratories (Zhang, Rajagopalan, Brown \& Wallace, 1997; Cooksey et al., 2008).

Most of the animal pathogens reported in scientific literature are members of the M. avium complex, such as M. avium subsp. paratuberculosis, the cause of severe gastroenteritis in ruminants, $M$. avium subsp. avium in birds and raptors, and M. avium subsp. hominissuis in swine (Aranaz et al., 2010). In captive elephants, M. avium is also commonly isolated (Payeur et al., 2002) but is rarely associated with disease (Yong et al., 2011). The only two species reported to be the cause of elephant morbidity and mortality are M. szulgai, isolated from an acute fatal disease in two African elephants kept in a London zoo (Lacasse et al., 2007), and M. elephantis from the lung abscess of an elephant that died of a chronic respiratory illness (Shojaei et al., 
91 2000). Generally, little is known about the epidemiology and zoonotic potential of NTMs among

92 animals in captivity.

93

94 While studying tuberculosis among captive Asian elephants in Malaysia (Ong et al., 2013), we

came across two strains of a slow-growing NTM that appeared to be identical in morphology and routine biochemical tests. We investigated the relationship between these two strains with conventional and whole genome sequence-based methods of strain differentiation. Our observations indicated two members of a possibly novel NTM species with highly similar genetic and protein profiles that characterize strains of clonal descent. Our whole genome sequence data also supported the reliability of conventional microbiological and molecular methods for the strain differentiation of non-tuberculous mycobacteria.

\section{Materials and Methods}

Bacterial isolation

UM_3 and UM_11 were isolated from two Asian elephants (Elephas maximus) in 2012. UM_3 was from the offspring of a Malaysian jungle elephant. It was born in 1997 and was raised in a conservation center $(\mathrm{KG})$ before its transfer to a zoo about $250 \mathrm{~km}$ away, in 2002 . UM_11 was isolated from a 71 year-old elephant acquired from Assam, India, and brought to KG in 2010 under the aegis of the Department of Wildlife and National Parks, Peninsular Malaysia. Hence,

110 both elephants had been in the same conservation center, eight years apart. Both had no overt

111 signs of disease at the time of sampling. The collection of elephant trunk wash was approved by

112 the Animal Care and Use Committee, Faculty of Veterinary Medicine, Universiti Putra Malaysia

113 (reference number: UPM/FPV/PS/3.2.1.551/AUP-R163) (Ong et al., 2013). 
115 Elephant trunk wash was collected using the "triple sample method", decontaminated with the

116 modified Petroff method, and further processed as described previously (Ong et al., 2013).

117 Mycobacterial isolation was carried out in the BACTEC Mycobacteria Growth Indicator Tube 118 (MGIT) 960 System (Becton Dickinson, USA). Positive BACTEC cultures were sub-cultured 119 onto Lowenstein-Jensen agar for incubation at $36^{\circ} \mathrm{C}$ in light and in the dark, for up to 8 weeks, to 120 observe growth rate and colonial morphology. The Tibilia test (Tibilia Genesis, Hangzhou, 121 China) for the detection of Mycobacterium tuberculosis-specific MPB64 antigen was performed 122 according to the manufacturer's instructions, to differentiate $M$. tuberculosis from non123 tuberculous mycobacteria (NTM). For further investigations, isolates were subcultured on 124 Middlebrook 7H10 agar with OADC supplement (BBL, Becton Dickinson).

Molecular identification of isolates

127 DNA was extracted by boiling a few loopfuls of each isolate suspended in sterile distilled water, 128 at $100^{\circ} \mathrm{C}$ for $30 \mathrm{~min}$, followed by centrifuging at $16,100 \mathrm{~g}$ for $2 \mathrm{~min}$. The supernatant was used 129 for a hsp65-based PCR (Telenti et al., 1993), using primers Tb11 (5'-ACC AAC GAT GGT GTG 130 TCC AT) and Tb12 (5'-CTT GTC GAA CCG CAT ACC CT) to amplify a 441bp fragment in the 131 gene. PCR products were purified using the QIAquick PCR Purification kit (QIAGEN, 132 Germany) and sent for Sanger sequencing. The resultant DNA sequences were searched against 133 the NCBI non-redundant nucleotide database using BLAST web server (Altschul et al., 1990). 134 The most probable species for each isolate was identified based on the nucleotide sequence 135 similarity with reference strains. 
138 For conventional biochemical tests (Kent, 1985; Babady \& Wengenack, 2012) and rapid

139 biochemical profiling using API strips (bioMerieux, France), the inoculum used was a 140 suspension of each isolate in normal saline, made up to McFarland 0.5 turbidity. For the Biolog 141 Phenotype Microarray analysis, a turbidimeter was used to check the turbidity of the suspension 142 and bacterial cells were added to achieve 81\% T (transmittance). Microplates PM1, PM2, PM9 143 and PM10 were used with the OmniLog system (Biolog, USA). These Microplates are 96-well 144 microtiter plates containing different kinds of compounds. PM1 and PM2 test for carbon145 utilization patterns while PM9 and PM10 test for tolerance to a wide range of osmolytes and pH 146 (http://www.biolog.com/pmMicrobialCells.html). M. tuberculosis H37Ra was used as the control 147 in all these tests.

Protein profiling with liquid chromatography-mass spectrometry (Q-TOF LC/MS)

150 The NTM cells were lysed and proteins were extracted using the ProteoSpin detergent-free total 151 protein isolation kit (Norgen Biotek, Canada) with the Halt protease and phosphatase inhibitors 152 cocktail (Thermo Scientific, USA) included. The lysates were subsequently treated with $10 \mathrm{mM}$ 153 dithiothreitol (DTT; Bio-Rad, USA) at $37^{\circ} \mathrm{C}$ for $10 \mathrm{~min}$ and alkylated with $55 \mathrm{mM}$ 154 iodoacetamide (IAA; Bio-Rad) for $30 \mathrm{~min}$ at room temperature. The proteins in the sample were 155 digested with 1:50 (trypsin: protein) of MS-grade Pierce trypsin protease (Thermo Scientific) at $15637^{\circ} \mathrm{C}$ overnight. The samples were desalted using a Pierce C-18 spin column (Thermo Scientific) 157 and dried to completeness in a refrigerated CentriVap centrifugal vacuum concentrator 158 (Labconco, USA) before mass spectrometry analysis. 
160 Tryptic peptides were analyzed on the 1260 Infinity HPLC-Chip/MS System (Agilent, USA).

161 The HPLC-Chip was the Large Capacity C18 Chip (G4240-6210), which comprised a $160 \mathrm{~nL}$ 162 enrichment column and a $150 \mathrm{~mm}$ analytical column. HPLC-grade water $(0.1 \%$ formic acid $)$ and 163 acetonitrile $(0.1 \%$ formic acid $)$ were used as mobile phases A and B respectively. HPLC-grade 164 acetonitrile and formic acid were procured from Friendemann Schmidt (Australia) and Sigma 165 (USA), respectively. The Nanoflow gradient (\%B) used was: $3 \%$ at $0 \mathrm{~min}, 3 \%$ at $5 \mathrm{~min}, 35 \%$ at $16660 \mathrm{~min}, 40 \%$ at $67 \mathrm{~min}, 60 \%$ at $85 \mathrm{~min}, 60 \%$ at $95 \mathrm{~min}, 3 \%$ at $105 \mathrm{~min}$; stop time: 120 minutes; 167 nanopump flow rate: $0.3 \mathrm{uL} / \mathrm{min}$; capillary pump gradient: $3 \% \mathrm{~B}$ isocratic; capillary pump flow: $1682.5 \mathrm{uL} / \mathrm{min}$; chip value position: enrichment at $95 \mathrm{~min}$. An Agilent 6540 Accurate-Mass Q-TOF 169 LC/MS System operated in positive ion mode was used for mass detection, applying the 170 following settings: capillary voltage : $1850 \mathrm{~V}$; drying gas flow: $5 \mathrm{~L} / \mathrm{min}$; drying gas temperature: $171250^{\circ} \mathrm{C}$; fragmenter: $175 \mathrm{~V}$; skimmer: $65 \mathrm{~V}$; acquisition mode: autoMS/MS; scan range: 125-1700 $172 \mathrm{~m} / \mathrm{z}(\mathrm{MS}), 50-1700 \mathrm{~m} / \mathrm{z}$ (MS/MS); acquisition rate: $15 \mathrm{spectra} / \mathrm{s}$ (MS), $12 \mathrm{spectra} / \mathrm{s}$ (MS/MS); 173 isolation width (MS/MS): narrow $(\sim 1.3 \mathrm{~m} / \mathrm{z}$ ); collision energy: -4.8 (offset) + 3.6 (slope); max. 174 precursors/ cycle: 15; active exclusion: enabled, exclude after one spectrum, release after 0.25 175 min; charge state preference: 2, 3 and >3, sorted by abundance only; reference mass: 299.294457 and $1221.990637 \mathrm{~m} / \mathrm{z}$ from continuous addition of trace amounts of methyl stearate and HP-1221 calibrant respectively into the ionization region. Reference mass correction was enabled. De novo sequencing was conducted with PEAKS Studio 7.0 (Bioinformatics Solutions Inc., Canada) with default parameters, except that: i) parent mass error tolerance was $1.5 \mathrm{Da}$, ii) fragment mass error tolerance was $0.5 \mathrm{Da}$, iii) trypsin as digestion enzyme, iv) carbamidomethylation

181 (+57.02 Da, C) as fixed modification, v) oxidation (+15.99 Da, M) as variable modification, vi) 182 maximum variable post-translation modification allowed per peptide was three and vii) 
183

184

185

186

187

188

189

190

191

192

193

194

195

196

197

198

199

200

201

202

203

204

205

Mycobacterium parascrofulaceum ATCC BAA-614 UniProtKB reference proteomes database

was used for identifications.

Repetitive sequence-based PCR typing (Rep-PCR) with the Diversilab System

Rep-PCR strain typing relies on the amplification of sequences between repetitive elements interspersed throughout the genome (Healy et al., 2005). For the typing of our strains, we used the automated Diversilab system from Spectral Genomics, Inc., Houston, Tex. Mycobacterial cells harvested from MGIT960 tubes were used for DNA extraction, using the Ultra Clean microbial DNA isolation kit (Mo Bio Laboratories, Inc, CA.), according to the manufacturer's instructions. The extracted DNA was evaluated in the Nanodrop ND-2000 spectrophotometer (Thermo Scientific, Wilminton, DE, USA) and diluted to $35 \mathrm{ng} / \mu$ l for use in the DiversiLab system. The PCR was run on thermal cycler (Applied Biosystems, Foster City, CA) using the following conditions: initial denaturation at $95{ }^{\circ} \mathrm{C}$ for $5 \mathrm{~min}$, followed by 35 cycles of denaturation at $95{ }^{\circ} \mathrm{C}$ for $30 \mathrm{~s}$, annealing at $66{ }^{\circ} \mathrm{C}$ for $45 \mathrm{~s}$, extension at $72{ }^{\circ} \mathrm{C}$ for $1 \mathrm{~min}$, and final extension at $72{ }^{\circ} \mathrm{C}$ for $5 \mathrm{~min}$. Each reaction constitutes $18.0 \mu \mathrm{l}$ of rep-PCR MM1, $2.5 \mu 1$ of GeneAmp 10 X PCR buffer, $2.0 \mu 1$ of Primer Mix, $0.5 \mu 1$ of AmpliTaq DNA Polymerase and 2 $\mu l$ of template DNA. The detection of fluorescent rep-PCR fingerprint was carried out on microfluidic chips (LabChip device, Caliper technologies, Inc) on an Agilent model 2100 Bioanalyzer (Agilent Technologies, Inc, Palo Alto, CA). The subsequent fingerprint data was analyzed on the web-based DiversiLab v3.4.4 software (Bacterial Barcodes). The Top match option was used to find the closest match to the available DiversiLab Mycobacterium database in terms of similarity values based on the Pearson correlation coefficient. According to the manufacturer's guidelines, isolates were considered "different" if they had $<95 \%$ similarity and $\geq 2$ band differences for 
206 homogeneous organisms or $\geq 3$ band differences for heterogeneous organisms; "similar" if there

207 was $<97 \%$ similarity and 1 band difference for homogeneous organisms or up to 2 band

208 differences for heterogeneous organisms and "indistinguishable" if there was $>95 \%$ similarity

209 and no banding differences, including no variation in intensities of individual bands (Shutt et al., 210 2005).

211

212 Whole genome sequencing and phylogenomic analyses

213 For each strain, a Nextera DNA sequencing library was prepared as described previously

214 (Ngeow et al., 2015). Shotgun sequencing was performed with the Illumina HiSeq 2000 system.

215 The sequencing reads generated were investigated using FastQC (fastqc) (Andrews, 2010) and

216 assembled with CLC Genomics Workbench v.7.0. Gene prediction was made using Prokaryotic

217 Dynamic Programming Genefinding Algorithm (PRODIGAL) Version 2.60 (Hyatt et al., 2010).

218 The predicted CDSs were annotated by homology search against the NCBI nr database

219 (ftp://ftp.ncbi.nlm.nih.gov/blast/db/). rRNA and tRNA were detected using RNAmmer 1.2

220 Server (Lagesen et al., 2007) and tRNAscan-SE 1.21 (Schattner, Brooks \& Lowe, 2005). Phage

221 elements were searched for using PHAST (Zhou et al., 2011).

222

223 The genomic data obtained was used to determine the degree of genetic relatedness between the 224 two strains. For this purpose, we drew a Venn diagram with homologous genes identified as 225 genes sharing $>50 \%$ protein sequence identity, and constructed a Maximum Likelihood (ML) 226 phylogenomic tree, using single copy orthologous CDSs generated from OrthoMCL (Chen et al., 227 2006), aligned with MUSCLE (Edgar, 2004) and reconstructed with 100 bootstrap replicates 228 using Phyml 3.0 (Guindon \& Gascuel, 2003). For comparison, 13 selected genomes were 

05-1390 (CP003347), M. tusciae JS617 (AGJJ02), and M. xenopi (JAOC01), b) a slow-growing photochromogen M. kansasii ATCC 12478 (NC022663), c) non-pigmented slow-growers $M$. avium 104 (NC008595), M. intracellulare ATCC 13950 (NC016946), M. colombiense CECT 3035 (AFVW02), M. genavense ATCC 51234 (JAGZ01) and M. smegmatis str.MC2155 (NC_008596) d) non-pigmented fast-growers M. indicus pranii MTCC 9506 (NC018612) and

M. septicum DSM 44393 (CBMJ02), and e) a rapid-growing schotochromogenic strain $M$. iranicum UM_TJL (AUWT01). Nocardia farcinica (NC_006361) was used as the out-group.

ANI and TETRA analyses

For the calculation of average nucleotide identity (ANI) values (Nei \& Li, 1979), we determined all conserved genes in the two genomes by whole-genome sequence comparisons using the BLAST algorithm. Genes were considered conserved when they had a BLAST match of at least $30 \%$ overall sequence identity and at least $70 \%$ identity in the aligned region. Generally, strains tetra-nucleotide usage patterns, we used TETRA (http://www.megx.net/tetra) (Teeling et al., 2004) to calculate the correlation coefficients of the tetra-nucleotide signatures in the two genomes. Correlation coefficient values above 0.99 suggest a high probability of two strains being from the same species. 
252 We compared the allelic variants in seven housekeeping genes (argh, cya, gnd, murC, purH, 253 rpob and pta) in UM_3 and UM_11. Full-length gene sequences retrieved from the two 254 annotated genomes were concatenated and aligned with MAFFT software 255 (http://mafft.cbrc.jp/alignment/server/). We compared fragment sizes of the seven loci and 256 looked for polymorphic sites and SNPs.

257

258

259

260

261

262

263

264

265

266

267

268

269

270

271

273

\section{Results}

\section{Morphology, biochemistry and PCR-sequencing}

Both UM_3 and UM_11 grew after 10-14 days of incubation at $36^{\circ} \mathrm{C}$ on LJ agar. The colonies were small, bright yellow, schotochromogenic, discrete and moist-looking (Supplementary Figure $1 \mathrm{~A}$ and $1 \mathrm{~B})$. They did not grow at $45^{\circ} \mathrm{C}$ or in $5 \% \mathrm{w} / \mathrm{v}$ sodium chloride but grew at $22^{\circ} \mathrm{C}$ and in the presence of streptomycin $2 \mathrm{mg} / \mathrm{L}$ (Supplementary Table 1). The bacilli were acid-fast with the Ziehl-Neelson stain and were negative for the MPB64 antigen in the Tibilia test for $M$. tuberculosis. PCR-sequencing of the hsp65 gene identified both strains as M. paracrofulaceum with $99 \%$ coverage and $96 \%$ sequence similarity.

Standard biochemical tests (Supplementary Table 1) showed both strains to be negative in iron uptake, niacin production, urea hydrolysis and oxidase tests, but strongly positive in the $68^{\circ} \mathrm{C}$ catalase and semi-quantitative catalase tests. Of a total of 87 tests in three API kits (API 20NE, API Coryne and API $50 \mathrm{CH}$ ), only seven (8.0\%) gave different results: the fermentation/assimilation of glucose, maltose, lactose, mannitol, $N$-acetyl-glucosamine, AMD starch and potassium 5-Keto-gluconate G. In the Biolog assays, of 384 substrates surveyed, 24 
$274(6.3 \%)$ differentiated the two strains. These differences mostly involved the utilization of organic 275 acids as carbon sources and the ability to grow in 3 to $6 \%$ sodium salts (data not shown).

277 Protein profiles

278 The LC/MS data yielded 495,933 MS scans and 156,008 MS/MS scans. After filtration, 4,089

279 peptide-spectrum matches were obtained and a total of 1,206 proteins were identified with $0.6 \%$

280 FDR. Among the proteins identified, the majority (1160/1206 or 96.2\%) were highly conserved

281 in both strains; only 46 were differentially expressed. Using the PEAKS label-free quantification

282 tool, 5 proteins had more than 2-fold higher expression in UM_3 compared to UM_11 (Figure

283 1). These proteins (Elongation factor $\mathrm{Tu}$ [tr|D5PC89|], $60 \mathrm{kDa}$ chaperonin [tr|D5PBK4|], DNA-

284 binding protein HU (tr|D5PIY7|], Cyclic nucleotide-binding domain protein [tr|D5P8V4|] and

285 transcription elongation factor Gre [tr|D5PFQ0|]) seem to be involved in transcription and

286 translation regulation. In addition, 22 metabolic proteins (14 were expressed in UM_3 and 8

287 were expressed in UM_11), 5 ribosomal proteins (UM_3) and 2 bacterial secretion proteins

288 (UM_11) were also uniquely expressed among these two strains by sequence homology search 289 tool (SPIDER) of the PEAKS software (Supplementary Figure 2). However, without further

290 functional analysis, we were unable to show correlation between the expression of these proteins 291 and our API and selected Biolog phenotyping test results.

Rep-PCR typing

294 Our rep-PCR typing results showed 98.8\% similarity between the two strains in both the 295 dendrogram (Figure 2) and the overlay (Figure 3). By the manufacturer's guidelines, the two 296 strains should be classified as almost indistinguishable, as they showed an identical banding 
297 pattern with differences seen only in the intensities of a few individual bands (Supplementary

298 Figure 3A and 3B).

299

300 Genomic analyses

301 The Illumina sequencing generated 5,236,492 reads assembled into 177 contigs for UM_3 (at an 302 average coverage of 72 times and N50 of 98,626bp) and 6,279,982 reads assembled into 275 303 contigs for UM_11 (at an average coverage of 59 times and N50 of 126,029bp). The number of 304 putative coding sequences (CDS) predicted was 4958 for UM_3 and 5022 for UM_11. Both draft 305 genomes contained 5.2Mbp with a $\mathrm{G}+\mathrm{C}$ composition of $68.8 \%$. By the best BLAST hit results, 306 $68.7 \%-70.21 \%$ of their coding sequences mapped towards $M$. parascrofulaceum. Each genome had one rRNA operon (5S, 16S and 23S), 52 tRNAs and two phage-related elements which were the phage T7 F exclusion suppressor FxsA and a phage-related replication protein.

The ANI values showed $99.98 \%$ nucleotide similarity between the two strains compared to $88.43 \%$ similarity between each strain and M. parascrofulaceum. The TETRA correlation coefficient was 0.99999 between both strains and 0.99666 (UM_3) to 0.99678 (UM_11) between the two strains and M. parascrofulaceum, suggesting that both strains belong to the same species that is distinct from M. parasrofulaceum (Supplementary Table 2).

In the orthologs-based phylogenomic tree, both UM_3 and UM_11 were clustered together next to $M$. parascrofulaceum, with a genomic distance of 0.0000 base substitutions between them (Figure 4). The Venn diagram (Figure 5) showed the two strains sharing 4752 of 4797 (99.1\%)

319 gene families in the core genome (Supplementary Table 3). In the MLS sequence analysis of 
320 seven housekeeping genes, all corresponding genes in both strains were of the same gene length

321 and had identical allele sequences except for the murC gene which showed two possible indels

322 (2bp and $97 \mathrm{bp}$ ) in the 5' end of the gene (Figure 6).

323

324

325

326

327

329

330

331

332

333

334

335

336

337

338

339

340

341

342

\section{Discussion}

As NTM species are ubiquitous in natural environments, isolates from elephant trunk wash are usually considered environmental saprophytes colonizing the trunk or contaminating trunk wash during sample collection. It is conceivable, however, that respiratory infections can occur via the inhalation of aerosols from contaminated feed and water sources, and cross-infection can occur among elephants, during trumpeting and trunk-spraying. In epidemiological investigations, the route of transmission is established by demonstrating the presence of the infecting strain in the suspected source (whether environmental or animal) and in the affected animals. The two NTM strains in our study shared multiple biological features that indicated a high probability that they were strains of clonal descent from a common source. To study this possibility, we attempted strain differentiation using a multiphasic approach, as conventional bacterial typing methods have not been adequately evaluated for most NTM species.

In addition to routine biochemistry, we demonstrated phenotypic similarity with metabolic and protein profiling. The Biolog Phenotype MicroArray system that allows bacterial profiling based on their ability to grow and respire on compounds serving as carbon, nitrogen, phosphorus or sulfur sources, has been shown to be able to distinguish both slowly growing mycobacteria such as $M$. tuberculosis and M. leprae, as well as rapidly growing NTMs such as M. smegmatis, $M$. fortuitum, M. chelonae and M. phlei (Rahman, Jaques III \& Daniels, 2008). For UM_3 and 
343 UM_11, the Biolog tests showed only a minor difference in energy source utilization and salt

344 tolerance. The comparable metabolic profiles of the two strains correlated well with their highly

345 conserved protein profiles obtained with LCMS, suggesting that they were functionally very

346 similar. However, the protein profiling could have been limited by the lack of a reference

347 proteome for our strains. We had to use M. parascrofulaceum ATCC BAA-614 as our reference,

348 a strain that showed the highest hsp65 sequence identity but only around $70 \%$ coding gene 349 sequence similarity with UM_3 and UM_11.

351 For genotyping, we used the Diversilab system which has been adapted for use on mycobacteria.

352 It was reported to show either equivalent or higher discriminatory power than that of IS6110353 RFLP for the typing of M. tuberculosis (Jang et al., 2011). Among the NTM, it has been used 354 effectively for the strain differentiation of the M. avium complex (Cangelosi et al., 2004) as well 355 as M. abscessus isolates (Zelazny et al., 2009). Going by the interpretation guidelines 356 recommended by the manufacturer, UM_3 and UM_11 which showed more than 98\% band 357 pattern similarity, were almost indistinguishable and could very well be descendants of the same 358 clone.

More comprehensive information for strain differentiation came from the comparison of whole 361 genome sequences that showed both strains to be identical in their genome size, $\mathrm{G}+\mathrm{C}$ content, 362 number and sequence of tRNAs, number and sequence of phage elements, ANI values and 363 tetranucleotide signatures, as well as $99 \%$ conservation in gene families. The larger number of

364 unique genes in UM_3, as shown in the Venn diagram, suggested that it might be the progenitor 
365 to UM_11 which appeared to have lost some genes. This observation ties in well with the history

366 of UM_3 being in the conservation center years before UM_11 was brought there.

367

368 We compared the sequences of seven housekeeping genes for both strains as MLS has been

369 shown to be not only reliable for outbreak investigations but also potentially useful for strain

370 identification and species designation of novel isolates (Bielaszewska et al., 2011). In the usual

371 application of MLS typing (MLST) for a bacterial species, each unique combination of locus

372 variants is assigned a sequence type (ST), which is compared to STs in a public MLST database

373 for that bacterial species. In our case, since our strains did not have a species designation, we

374 could not assign a definite ST to each strain; instead, we compared the allele profiles generated

375 by concatenating the seven gene sequences together for the comparison of sequence length and

376 variation. The almost identical profiles obtained for UM_3 and UM_11 are consistent with the

377 high genetic similarity demonstrated by the rep-PCR typing and the assumption of clonal 378 descent.

380 Unlike bacterial species differentiation that is guided by differences in 16S RNA sequences and 381 percentage DNA-DNA hybridization, there is no consensus guideline for bacterial strain 382 differentiation. In genotyping, molecular markers are used to generate distinct DNA fingerprints. 383 These markers are conserved alleles that have remained stable over much of evolution (Owen \& 384 Xerry, 2003). Unfortunately, the discriminatory power of commonly-used molecular markers has 385 not been adequately studied for most NTMs, especially highly conserved species with members 386 sharing very similar genetic fingerprints. Furthermore, some widely-prevalent genotypes can 387 become predominant in an environment. This situation can give rise to incorrect interpretation of 388 clonality among strains belonging to the same genotype. For these reasons, in an epidemiological 
389 investigation, strains showing similar DNA fingerprints cannot be interpreted to be clonally-

390 related unless an epidemiological link is established as well.

392 In our study, the history of a shared habitat for our two elephant hosts allowed us to assume a 393 clonal descent for the almost indistinguishable UM_3 and UM_11. The minor differences in their

394 gene expression could be due to their individual responses to environmental stimuli, and their 395 minor genetic differences could be the result of genetic drift in the course of their continuous 396 microevolution (Israel et al., 2001). Overall, our findings showed consistency in results from 397 different types of analysis and supported the reliability of current biological and molecular strain 398 differentiation methods for NTMs.

\section{Conclusion}

401 402 403 404 405 406 407

Strain differentiation with multiple approaches can potentially unveil important phenotypic and genotypic features worthy of further investigations. In our two closely related NTM strains, we showed differentially expressed proteins that could be involved in the regulation of transcription and translation as well as other metabolic and secretory activities. Downstream investigations might lead to the elucidation of gene regulation mechanisms that affect the appearance, survival and behavior of mycobacterial spp.

Our use of whole genome sequencing demonstrated obvious advantages of this approach over conventional genotyping. From the whole genome backbone, multiple nucleotide and gene sequences can be extracted and used in diverse ways to provide information for taxonomic classification, strain differentiation and the inference of evolutionary relationships. Nevertheless, 
412 more genetic studies on NTMs from different sources, geographic areas and times would help the

413 development of more reliable genotyping methods applicable to all NTM species, without the

414 need for species-specific modifications.

415

416

Nucleotide sequence accession numbers

417 The whole-genome sequences have been deposited in GenBank: LATB00000000

418 (Mycobacterium sp. UM_3) and LATC00000000 (Mycobacterium sp. UM_11).

419

420

421

422

423

424

425

426

427

428

429

430

431

432

433

434

435

436

437

438

439

440

References

Altschul SF, Gish W, Miller W, Myers EW, Lipman DJ. 1990. Basic local alignment search tool. Journal of Molecular Biology 215:403-410.

Andrews S. 2010. FastQC: A Quality Control tool for High Throughput Sequence Data. http://www.bioinformatics.babraham.ac.uk/projects/fastqc/

Aranaz A., Bezos J., Romero B., Castellanos E., Rodriguez-Campos S., Casal C., Fernandez de Mera IG., Lozano F., Moya N., de Juan L. MA and DL. 2010. The role of Mycobacteria in Veterinary Medicine. 31st Annual Congress of the European Society of Mycobacteriology (ESM 2010), European Society of Mycobacteriology, Bled, Slovenia, July 5th, 2010.

Babady NE, Wengenack NL. 2012. Understanding Tuberculosis - Global Experiences and Innovative Approaches to the Diagnosis. InTech.

Bielaszewska M, Mellmann A, Zhang W, Köck R, Fruth A, Bauwens A, Peters G, Karch H. 2011. Characterisation of the Escherichia coli strain associated with an outbreak of haemolytic uraemic syndrome in Germany, 2011: a microbiological study. The Lancet. Infectious Diseases 11:671-676.

Cangelosi GA, Freeman RJ, Lewis KN, Livingston-Rosanoff D, Shah KS, Milan SJ, Goldberg S V. 2004. Evaluation of a High-Throughput Repetitive-Sequence-Based PCR System for DNA Fingerprinting of Mycobacterium tuberculosis and Mycobacterium avium Complex Strains. Journal of Clinical Microbiology 42:2685-2693.

Chen F, Mackey AJ, Stoeckert CJ, Roos DS. 2006. OrthoMCL-DB: querying a comprehensive multi-species collection of ortholog groups. Nucleic Acids Research 34:D363-368. 
441

442

443

444

445

446

447

448

449

450

451

452

453

454

455

456

457

458

459

460

461

462

463

464

465

466

467

468

469

470

471

472

473

474

475

Cooksey RC, Jhung MA, Yakrus MA, Butler WR, Adékambi T, Morlock GP, Williams M, Shams AM, Jensen BJ, Morey RE, Charles N, Toney SR, Jost KC, Dunbar DF, Bennett V, Kuan M, Srinivasan A. 2008. Multiphasic approach reveals genetic diversity of environmental and patient isolates of Mycobacterium mucogenicum and Mycobacterium phocaicum associated with an outbreak of bacteremias at a Texas hospital. Applied and Environmental Microbiology 74:2480-2387.

Edgar RC. 2004. MUSCLE: multiple sequence alignment with high accuracy and high throughput. Nucleic Acids Research 32:1792-1797.

Gori A, Bandera A, Marchetti G, Esposti AD, Catozzi L, Nardi GP, Gazzola L, Ferrario G, van Embden JDA, van Soolingen D, Moroni M, Franzetti F. 2005. Spoligotyping and Mycobacterium tuberculosis. Emerging Infectious Diseases 11:1242-1248.

Guindon S, Gascuel O. 2003. A simple, fast, and accurate algorithm to estimate large phylogenies by maximum likelihood. Systematic Biology 52:696-704.

Healy M, Huong J, Bittner T, Lising M, Frye S, Raza S, Schrock R, Manry J, Renwick A, Nieto R, Woods C, Versalovic J, Lupski JR. 2005. Microbial DNA typing by automated repetitive-sequence-based PCR. Journal of Clinical Microbiology 43:199-207.

Hyatt D, Chen G-L, Locascio PF, Land ML, Larimer FW, Hauser LJ. 2010. Prodigal: prokaryotic gene recognition and translation initiation site identification. $B M C$ Bioinformatics 11:119.

Israel DA, Salama N, Krishna U, Rieger UM, Atherton JC, Falkow S, Peek RM. 2001. Helicobacter pylori genetic diversity within the gastric niche of a single human host. Proceedings of the National Academy of Sciences of the United States of America 98:14625-14630.

Jagielski T, Jakko van Ingen J, Rastogi N, Dziadek J, Mazur PK, Bielecki J. 2014. Current Methods in the Molecular Typing of Mycobacterium tuberculosis and Other Mycobacteria. BioMed Research International 2014:21

Jang MH, Choi GE, Shin B-M, Lee SH, Kim S-R, Chang CL, Kim J-M. 2011. Comparison of an automated repetitive sequence-based PCR microbial typing system with IS6110-restriction fragment length polymorphism for epidemiologic investigation of clinical Mycobacterium tuberculosis isolates in Korea. The Korean Journal of Laboratory Medicine 31:282-284.

Kent PT. 1985. Public health mycobacteriology: a guide for the level III laboratory. Atlanta, Ga: U.S. Dept. of Health and Human Services, Public Health Service, Centers for Disease Control.

Lacasse C, Terio K, Kinsel MJ, Farina LL, Travis DA, Greenwald R, Lyashchenko KP, Miller M, Gamble KC. 2007. Two cases of atypical mycobacteriosis caused by Mycobacterium 
476

szulgai associated with mortality in captive African elephants (Loxodonta africana). Journal of Zoo and Wildlife Medicine 38:101-107.

Lagesen K, Hallin P, Rødland EA, Staerfeldt H-H, Rognes T, Ussery DW. 2007. RNAmmer: consistent and rapid annotation of ribosomal RNA genes. Nucleic Acids Research 35:31003108 .

Lavender CJ, Stinear TP, Johnson PD, Azuolas J, Benbow ME, Wallace JR, Fyfe JA. 2008. Evaluation of VNTR typing for the identification of Mycobacterium ulcerans in environmental samples from Victoria, Australia. FEMS Microbiol Lett. 287:250-255.

Mijs W, de Haas P, Rossau R, Van der Laan T, Rigouts L, Portaels F, van Soolingen D. 2002. Molecular evidence to support a proposal to reserve the designation "Mycobacterium avium subsp. avium" for bird-type isolates and " $M$. avium sub sp. hominissuis" for the human/porcine type of M. avium. International Journal of Systematic and Evolutionary Microbiology 52:1505-1518.

Nei M, Li WH. 1979. Mathematical model for studying genetic variation in terms of restriction endonucleases. Proceedings of the National Academy of Sciences 76:5269-5273.

Ngeow YF, Wong YL, Tan JL, Hong KW, Ng HF, Ong BL, Chan KG. 2015. Identification of New Genomospecies in the Mycobacterium terrae Complex. PLOS ONE 10:e120789.

Ong BL, Ngeow YF, Razak MFAA, Yakubu Y, Zakaria Z, Mutalib AR, Hassan L, Ng HF, Verasahib K. 2013. Tuberculosis in captive Asian elephants (Elephas maximus) in Peninsular Malaysia. Epidemiology and Infection 141:1481-1487.

Owen RJ, Xerry J. 2003. Tracing clonality of Helicobacter pylori infecting family members from analysis of DNA sequences of three housekeeping genes (ureI, atpA and ahpC), deduced amino acid sequences, and pathogenicity-associated markers (cagA and vacA). Journal of Medical Microbiology 52:515-524.

Payeur JB, Jarnagin JL, Marquardt JG, Whipple DL. 2002. Mycobacterial isolations in captive elephants in the United States. Annals of the New York Academy of Sciences 969:256-258.

Rahman M, Jaques III S, Daniels L. 2008. Carbon source utilization pattern among rapidly growing Mycobacterium (RGM) species by phenotypic array analysis using Biolog OmniLog Systems. In: SC-ASM Conference. Austin, Texas,.

Schattner P, Brooks AN, Lowe TM. 2005. The tRNAscan-SE, snoscan and snoGPS web servers for the detection of tRNAs and snoRNAs. Nucleic Acids Research 33:W686-689.

Shojaei H, Magee JG, Freeman R, Yates M, Horadagoda NU, Goodfellow M. 2000. Mycobacterium elephantis sp. nov., a rapidly growing non-chromogenic Mycobacterium isolated from an elephant. International Journal of Systematic and Evolutionary Microbiology 50 Pt 5:1817-1820. 
Shutt CK, Pounder JI, Page SR, Schaecher BJ, Woods GL. 2005. Clinical evaluation of the DiversiLab microbial typing system using repetitive-sequence-based PCR for characterization of Staphylococcus aureus strains. Journal of Clinical Microbiology 43:1187-1192.

Teeling H, Waldmann J, Lombardot T, Bauer M, Glöckner FO. 2004. TETRA: a web-service and a stand-alone program for the analysis and comparison of tetranucleotide usage patterns in DNA sequences. BMC Bioinformatics 5:163.

Telenti A, Marchesi F, Balz M, Bally F, Böttger EC, Bodmer T. 1993. Rapid identification of mycobacteria to the species level by polymerase chain reaction and restriction enzyme analysis. Journal of Clinical Microbiology 31:175-178.

Thibault VC, Grayon M, Boschiroli L, Hubbans C, Overduin P, Stevenson K, Gutierrez MC, Supply P, Biet F. 2007. New variable numbertandem-repeat markers for typing Mycobacterium avium subsp. paratuberculosis and $M$. avium strains: comparison with IS900 and IS1245 restriction fragment length polymorphism typing. Journal of Clinical Microbiology 45:2404-2410.

Yong H, Choi G-E, Lee BS, Whang J, Shin SJ. 2011. Disseminated infection due to Mycobacterium avium subsp. avium in an Asian elephant (Elephas maximus). Journal of Zoo and Wildlife Medicine 42:743-746.

Zelazny AM, Root JM, Shea YR, Colombo RE, Shamputa IC, Stock F, Conlan S, McNulty S, Brown-Elliott BA, Wallace RJ, Olivier KN, Holland SM, Sampaio EP. 2009. Cohort study of molecular identification and typing of Mycobacterium abscessus, Mycobacterium massiliense, and Mycobacterium bolletii. Journal of Clinical Microbiology 47:1985-1995.

Zhang Y, Rajagopalan M, Brown BA, Wallace RJ Jr. 1997. Randomly amplified polymorphic DNA PCR for comparison of Mycobacterium abscessus strains from nosocomial outbreaks. Journal of ClinicalMicrobiology. 35:3132-3139.

Zhou Y, Liang Y, Lynch KH, Dennis JJ, Wishart DS. 2011. PHAST: a fast phage search tool. Nucleic Acids Research 39:W347-352. 
539

540

541

542

543

544

545

546

547

548

549

550

551

552

553

554 555 557

556 Figure 6. Alignment of murC gene sequences in UM_3 (396bp) and UM_11 (495 bp)

\section{Figures}

Figure 1. Hit map of differential protein profile of UM_3 and UM_11 generated by PEAKS Studio 7.0. Tr|D5PIY7|: DNA-binding protein HU; tr|D5PBK4|: 60 kDa chaperonin; tr|D5P8V4|: cyclic nucleotide-binding domain protein; tr|D5PC89|: elongation factor Tu and tr|D5PFQ0|: transcription elongation factor Gre.

Figure 2. Dendrogram of UM_3 and UM_11 with other Mycobacterium isolates in the DiversiLab Mycobacterium database. UM_3 and UM_11 are $98.8 \%$ similar.

Figure 3. Overlay of rep-PCR fingerprints of UM_3 and UM_11.

Figure 4. Orthologs-based phylogenetic tree showing UM_3 and UM_11 in relation to other mycobacterial spp. Nocardia farcinica NC0063 is the out-group.

Figure 5. Venn diagram showing sharing of gene families between UM_3 and UM_11 


\section{Supplementary Information}

559

560 Supplementary Table 1. Results of conventional and API tests for two NTM strains and M.

561 tuberculosis H37Ra control

562 pos, positive; neg, negative; nd, not done;+/-, weak positive

563

564

Supplementary Table 2. ANI and TETRA results for UM_3, UM_11 and M. parascrofulaceum

a) ANI results for UM_3, UM_11 and M. parascrofulaceum

ADNVOI, M. paracrofulaceum

$567 *$ Numbers indicate \% similarity in 4799 shared genes

568

569

b) TETRA results for UM_3, UM_11 and M. parascrofulaceum

ADNVOI, $M$. paracrofulaceum

571

*Numbers indicate correlation coefficient values

572

573

Supplementary Table 3. Strain-specific genes in UM_3 and UM_11

574

575 Supplementary Figure 1. Colonies of (A) UM_3 and (B) UM_11 subcultured on Middlebrook $5767 \mathrm{H} 10$ agar with OADC supplement.

577

578 Supplementary Figure 2. Hit map of unique protein profile of UM_3 and UM_11 generated by PEAKS Studion 7.0 sequence homology search (SPIDER) tool.

580

581 Supplementary Figure 3. Diversilab report on (A) UM_3 (AF3) and (B) UM_11 (Kg11)

582 showing percentage similarity with other mycobacterium spp. 
1

Hit map of differential protein profile of UM_3 and UM_11 generated by PEAKS Studio 7.0 .

Tr|D5PIY7|: DNA-binding protein HU; tr|D5PBK4|: 60 kDa chaperonin; tr|D5P8V4|: cyclic nucleotide-binding domain protein; $\operatorname{tr|D5PC89|:~elongation~factor~Tu~and~tr|D5PFQ0|:~}$ transcription elongation factor Gre.

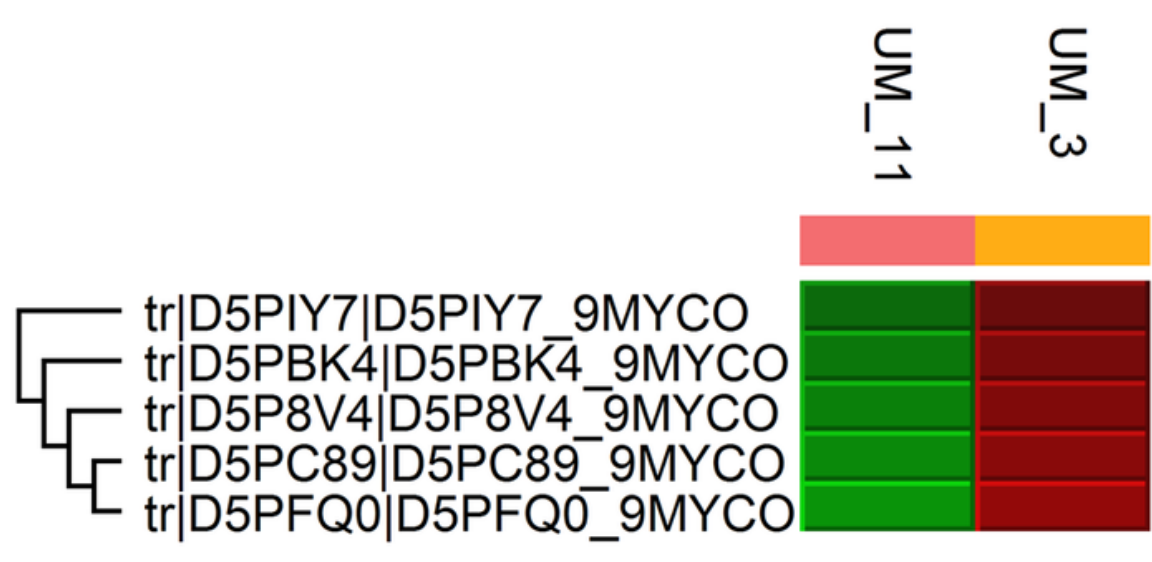

$$
\log 2 \text { (ratio) }
$$

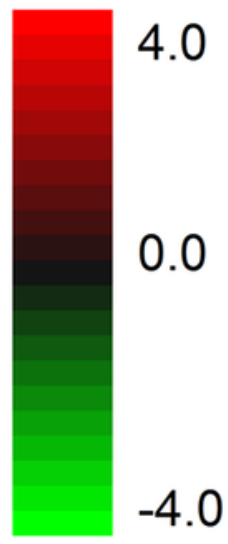

UM_11

UM_3 


\section{2}

Dendrogram of UM_3 and UM_11 with other Mycobacterium isolates in the DiversiLab Mycobacterium database.

UM_3 and UM_11 are $98.8 \%$ similar.
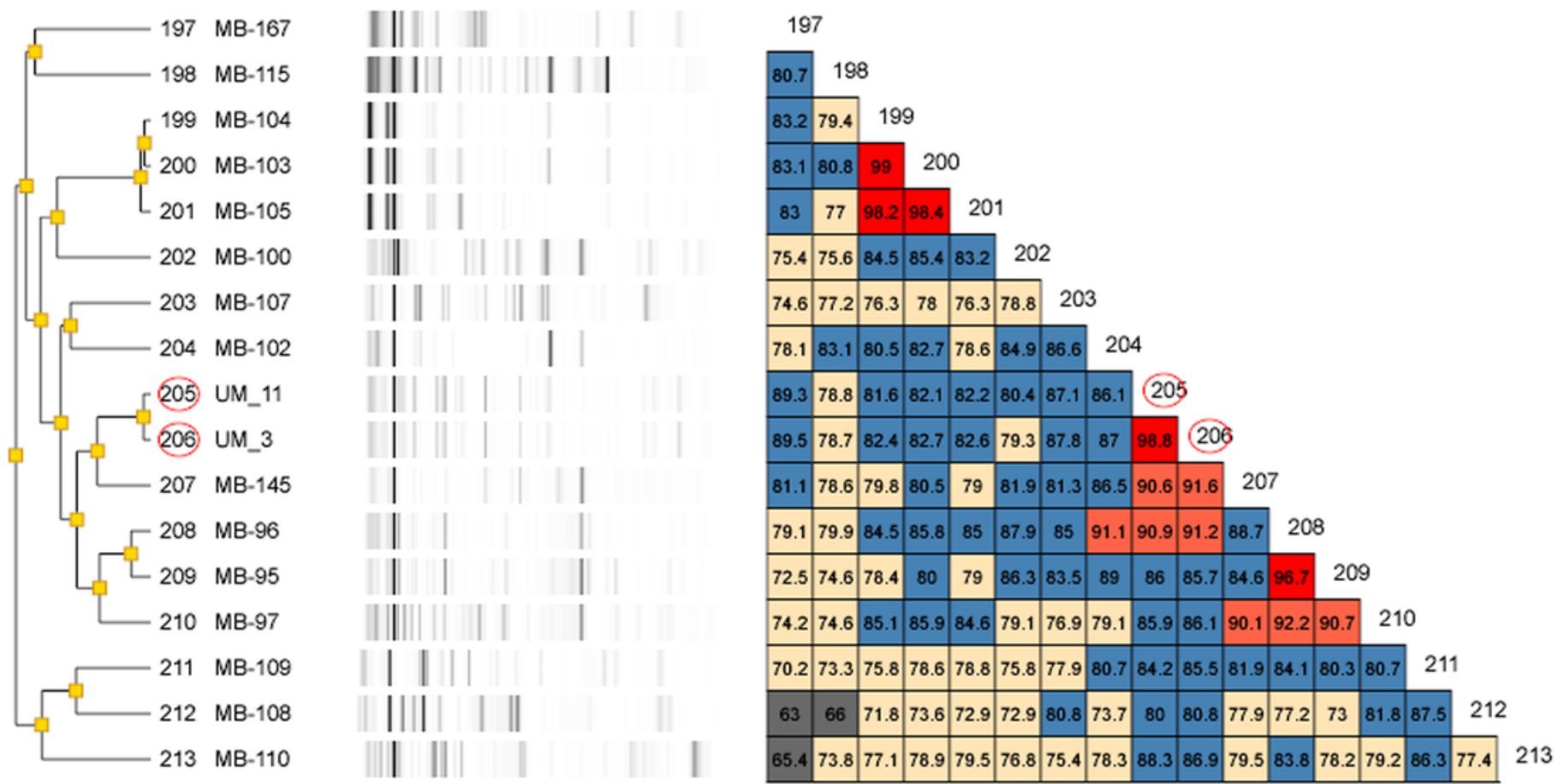
3

Overlay of rep-PCR fingerprints of UM_3 and UM_11.

Diversilab v3.4

PC

N91
Similarity: $98.8 \%$

$\begin{array}{ll}\text { Key } & \text { Sample ID } \\ 205 & \text { UM_11 } \\ 206 & \text { UM_3 }\end{array}$

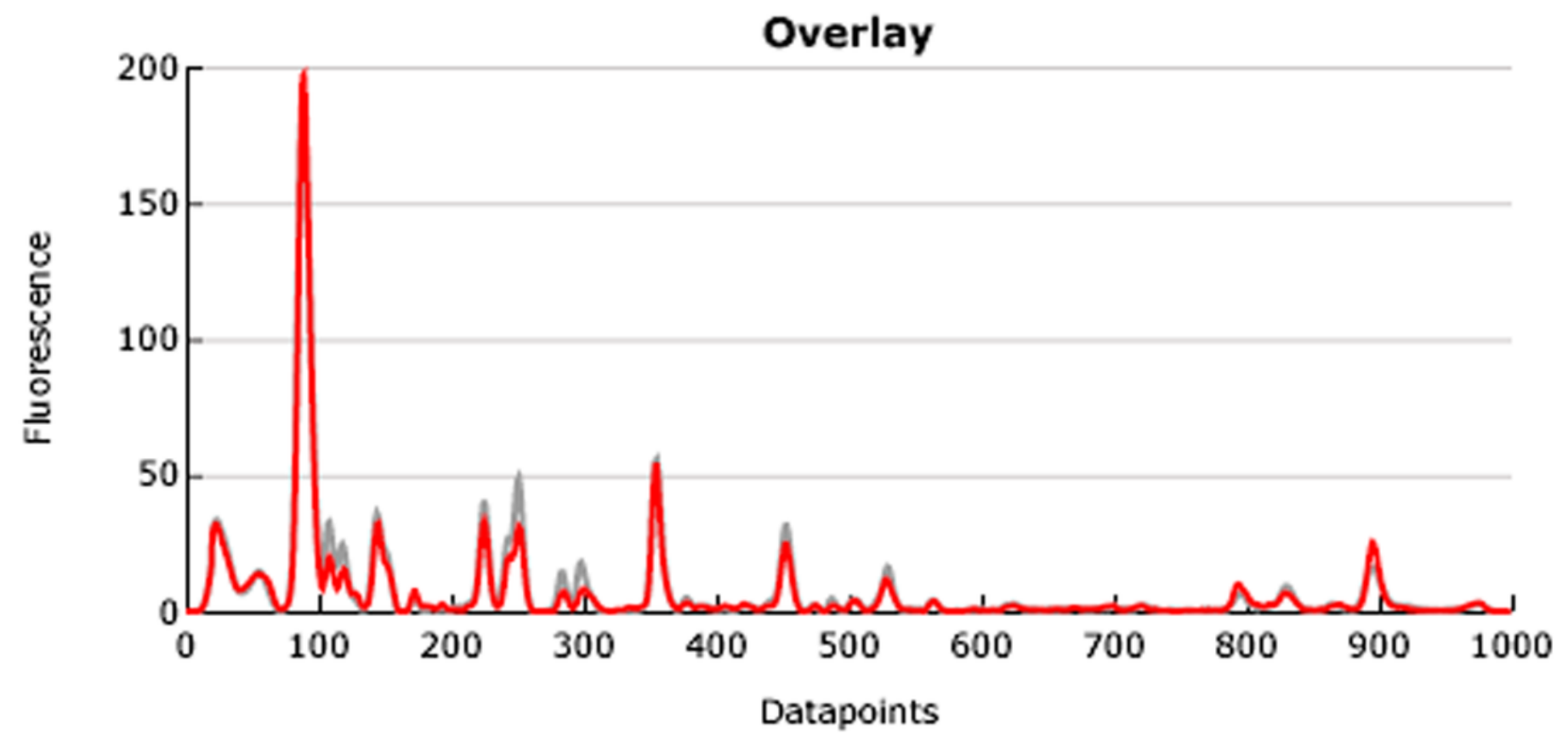


4

Orthologs-based phylogenetic tree showing UM_3 and UM_11 in relation to other mycobacterial spp.

Nocardia farcinica NC0063 is the out-group.

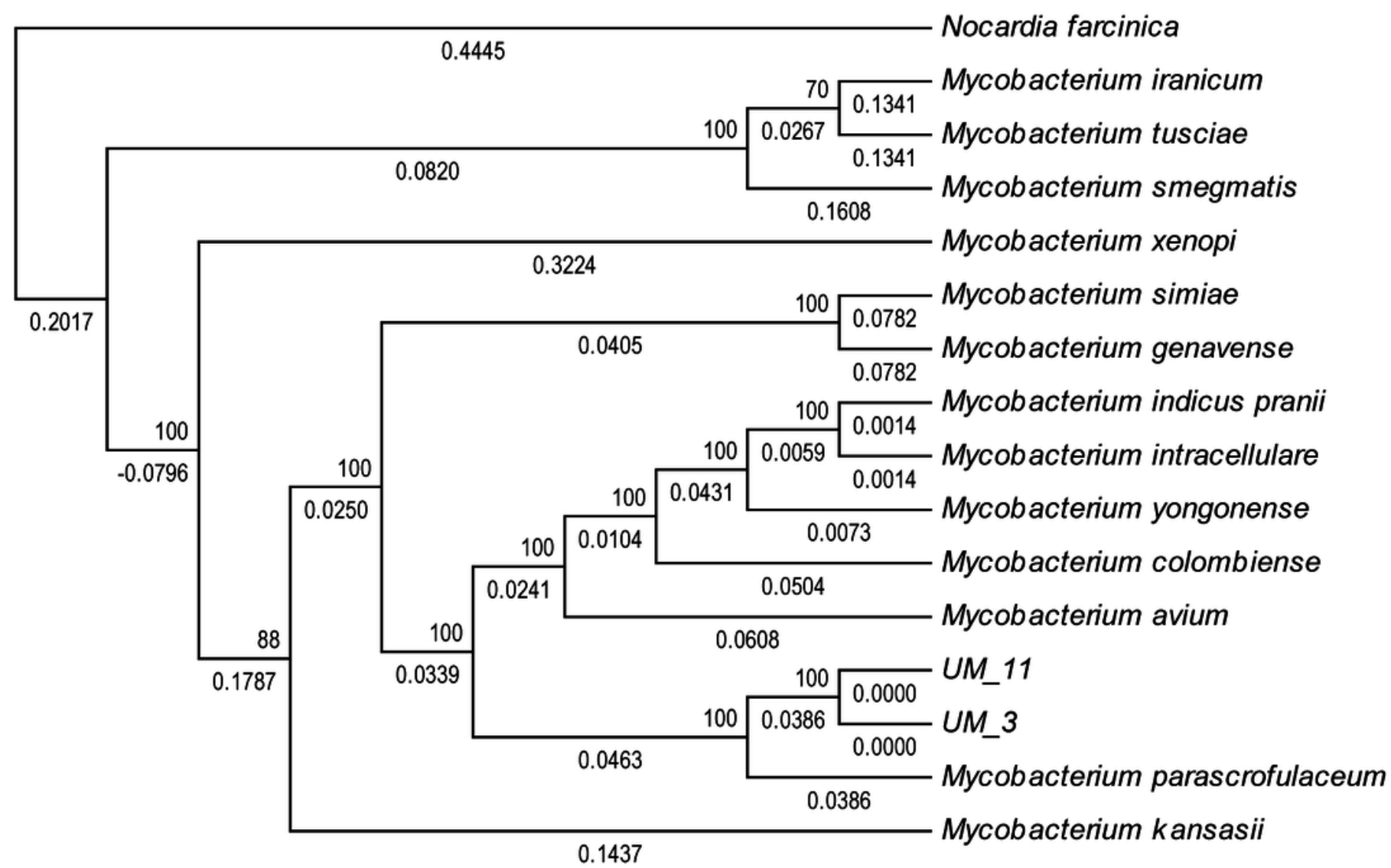


5

Venn diagram showing sharing of gene families between UM_3 and UM_11

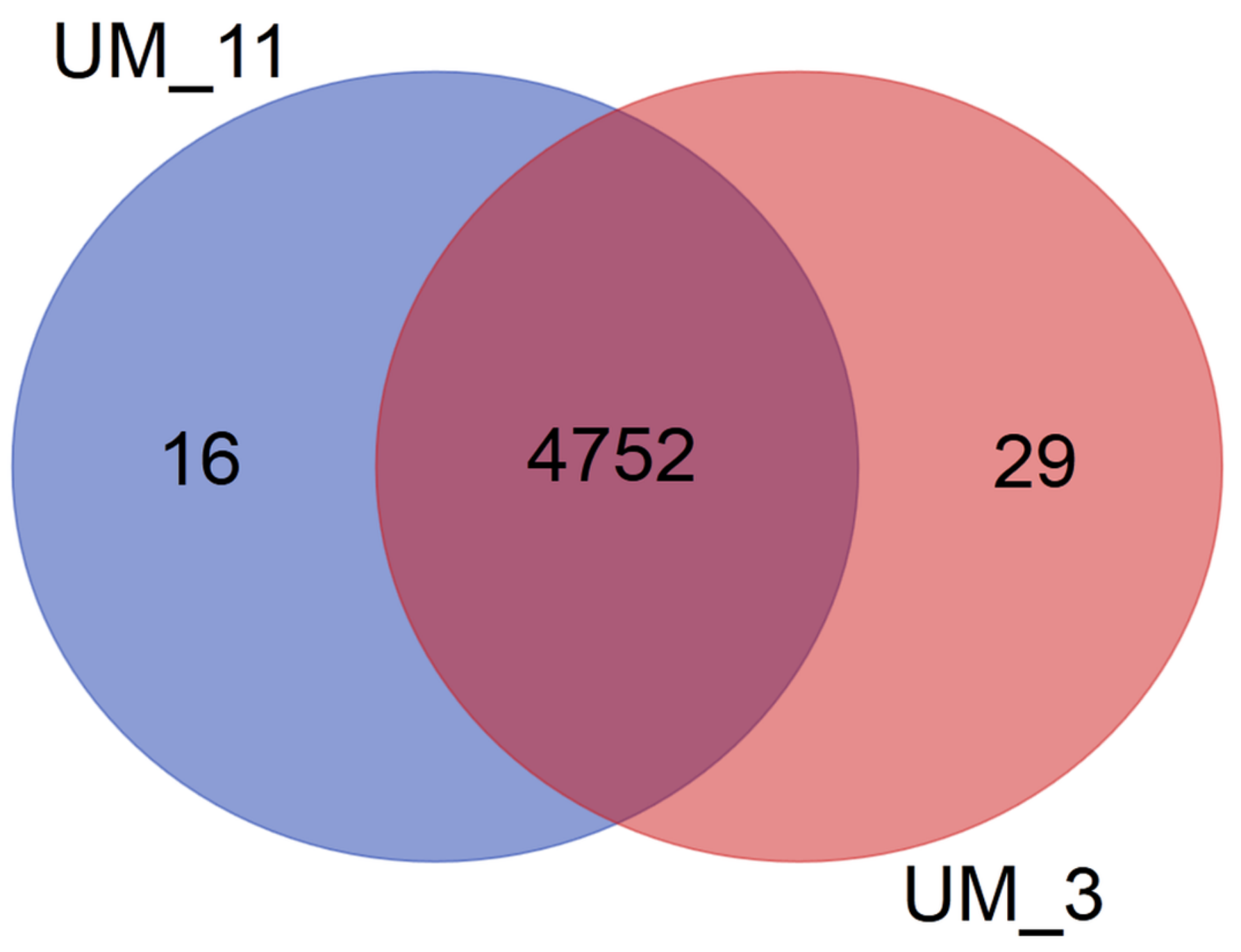


6

\section{Alignment of murC gene sequences in UM_3 (396bp) and UM_11 (495 bp)}

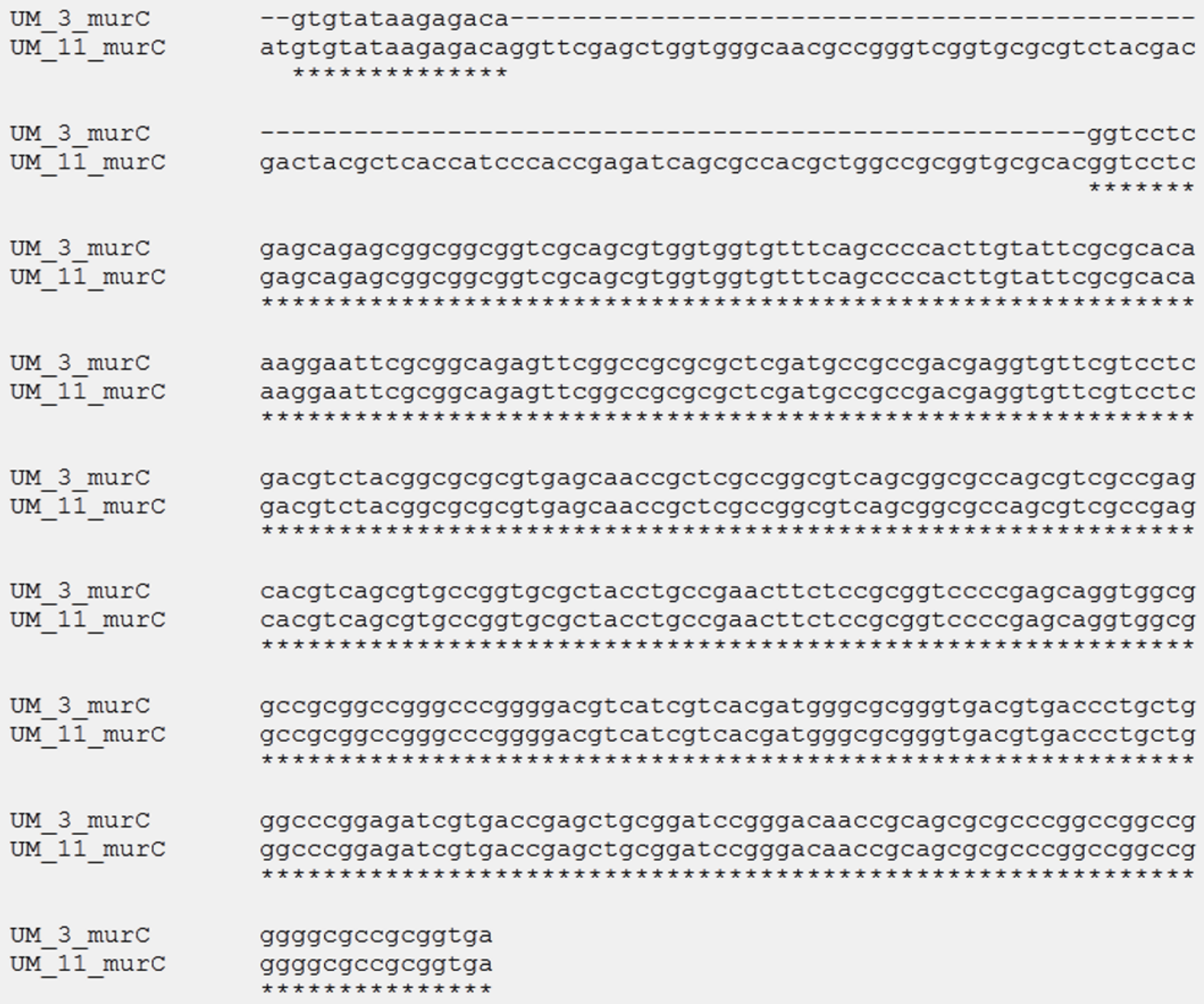

